\title{
DIRECT PRODUCTS OF AUTOMATIC SEMIGROUPS
}

\author{
C. M. CAMPBELL, E. F. ROBERTSON, N. RUŠKUC and R. M. THOMAS
}

(Received 3 October 1998; revised 21 December 1999)

Communicated by D. Easdown

\begin{abstract}
It is known that the direct product of two automatic groups is automatic. The notion of automaticity has been extended to semigroups, and this result for groups has been generalized to automatic monoids. However, the direct product of two automatic semigroups need not be finitely generated and hence not automatic.

Robertson, Ruškuc and Wiegold have determined necessary and sufficient conditions for the direct product of two finitely generated semigroups to be finitely generated. Building on this, we prove the following. Let $S$ and $T$ be automatic semigroups; if $S$ and $T$ are infinite, then $S \times T$ is automatic if and only if $S^{2}=S$ and $T^{2}=T$; if $S$ is finite and $T$ is infinite, then $S \times T$ is automatic if and only if $S^{2}=S$. As a consequence, we have that, if $S$ and $T$-are automatic semigroups, then $S \times T$ is automatic if and only if $S \times T$ is finitely generated.
\end{abstract}

2000 Mathematics subject classification: primary 20M05, $20 \mathrm{M} 35$.

\section{Introduction}

It is well known that the direct product of two automatic groups is automatic (see [3] for example). In [2] the notion of automaticity was extended to semigroups, and it was shown that the direct product of two automatic monoids is automatic. However, the direct product of two automatic semigroups need not be finitely generated $(\mathbb{N} \times \mathbb{N}$ for example) and hence not automatic. In [6] it was shown that, if $S$ and $T$ are finitely generated infinite semigroups, then $S \times T$ is finitely generated if and only if $S^{2}=S$ and $T^{2}=T$, whereas, if $S$ is a finite semigroup and $T$ is a finitely generated infinite semigroup, then $S \times T$ is finitely generated if and only if $S^{2}=S$. (The direct product of two finite semigroups is finite, and hence clearly finitely generated.) Given this we prove:

(C) 2000 Australian Mathematical Society 0263-61 15/2000\$A2.00+0.00 
THEOREM 1.1. Let $S$ and $T$ be automatic semigroups.

(i) If $S$ and $T$ are infinite, then $S \times T$ is automatic if and only if $S^{2}=S$ and $T^{2}=T$.

(ii) If $S$ is finite and $T$ is infinite, then $S \times T$ is automatic if and only if $S^{2}=S$.

Of course, if both $S$ and $T$ are finite, then $S \times T$ is finite, and hence automatic. We prove part (i) of Theorem 1.1 in Section 3, and part (ii) in Section 4.

Combining Theorem 1.1 with [6], we have the following consequence.

COROLlaRY 1.2. Let $S$ and $T$ be automatic semigroups. Then $S \times T$ is automatic if and only if $S \times T$ is finitely generated.

Unlike automatic groups, automatic semigroups need not be finitely presented, as is shown in [2, Example 4.4]. Combining Corollary 1.2 with [6, Example 8.4], we obtain an infinite collection of such examples.

\section{Definitions}

For any finite set $A$, let $A^{+}$denote the set of aH non-empty words over $A$, and let $A^{*}$ denote the set of all words over $A$ (including the empty word $\epsilon$ ). If $A$ is a set of generators of a semigroup $S$, then there is a natural homomorphism $\theta: A^{+} \rightarrow S$, where each word $\alpha$ of $A^{+}$is mapped to the corresponding element of $S$. Usually we suppress the reference to $\theta$, writing $\alpha$ for the element of the semigroup represented by the word $\alpha$.

As with automatic groups, we define a mapping $\delta_{A}: A^{*} \times A^{*} \rightarrow A(2, \$)^{*}$, where $\$ \notin A$ and $A(2, \$)=((A \cup\{\$\}) \times(A \cup\{\$\}))-\{(\$, \$)\}$, by

$$
\left(a_{1} \cdots a_{n}, b_{1} \cdots b_{m}\right) \delta_{A}= \begin{cases}\left(a_{1}, b_{1}\right) \cdots\left(a_{n}, b_{n}\right) & \text { if } n=m \\ \left(a_{1}, b_{1}\right) \cdots\left(a_{n}, b_{n}\right)\left(\$, b_{n+1}\right) \cdots\left(\$, b_{m}\right) & \text { if } n<m \\ \left(a_{1}, b_{1}\right) \cdots\left(a_{m}, b_{m}\right)\left(a_{m+1}, \$\right) \cdots\left(a_{n}, \$\right) & \text { if } n>m .\end{cases}
$$

If $S$ is a semigroup, $A$ a finite generating set, $L$ a regular subset of $A^{+}$, and $\phi: A^{+} \rightarrow S$ a homomorphism with $L \phi=S$, we say that $(A, L)$ is an automatic structure for $S$ if the sets

$$
\begin{aligned}
& L_{=}=\{(\alpha, \beta): \alpha, \beta \in L, \alpha=\beta \text { in } S\} \delta_{A} \quad \text { and } \\
& L_{a}=\{(\alpha, \beta): \alpha, \beta \in L, \alpha a=\beta \text { in } S\} \delta_{A} \quad(a \in A)
\end{aligned}
$$

are regular in $A(2, \$)^{*}$. If a semigroup $S$ has an automatic structure $(A, L)$ for some $A$ and $L$, then we say that $S$ is automatic. Note that a semigroup may have an automatic 
structure with respect to one finite generating set but not with respect to another (see [2, Example 4.5]), although this does not happen in groups (see [3]) or monoids (see [4]). For properties of regular sets see [5].

\section{Two infinite factors}

In this section we prove part (i) of Theorem 1.1. By [6, Theorem 2.1], if $S^{2} \neq S$ or $T^{2} \neq T$, then $S \times T$ is not finitely generated, and hence cannot be automatic. For the remainder of this section $S$ and $T$ are infinite automatic semigroups with $S^{2}=S$ and $T^{2}=T$.

We let $(A, L)$ be an automatic structure of $S$, where

$$
A=\left\{a_{1}, \ldots, a_{m}\right\} .
$$

Since $S^{2}=S$, there exists a function $\eta:\{1, \ldots, m\} \rightarrow\{1, \ldots, m\}$ and words $\zeta_{1}, \ldots, \zeta_{m} \in A^{+}$such that

$$
a_{i}=a_{i \eta} \zeta_{i}
$$

for each $i$. Introduce a new alphabet

$$
A^{\prime}=\left\{a_{1}, \ldots, a_{m}, a_{1}^{\prime}, \ldots, a_{m}^{\prime}\right\},
$$

where $a_{i}^{\prime}$ represents the element $\zeta_{i}$. Let

$$
W=\left\{a_{i_{1}} \cdots a_{i_{k}} a_{i_{k+1}}^{\prime} \cdots a_{i_{k+1}}^{\prime}: k \geq \hat{1}, l \geq 0, i_{k+p}=i_{k+p+1} \eta(0 \leq p \leq l-1)\right\},
$$

and let

$$
L^{\prime}=\left\{a_{i_{1}} \cdots a_{i_{k}} a_{i_{k+1}}^{\prime} \cdots a_{i_{k+1}}^{\prime} \in W: a_{i_{1}} \cdots a_{i_{k-1}} a_{i_{k+1}} \in L\right\}
$$

Define a mapping $\theta: W \rightarrow A^{+}$by

$$
\left(a_{i_{1}} \cdots a_{i_{k}} a_{i_{k+1}}^{\prime} \cdots a_{i_{k+1}}^{\prime}\right) \theta=a_{i_{1}} \cdots a_{i_{k-1}} a_{i_{k+1}} .
$$

Note that $w=w \theta$ holds in $S$ for every $w \in W$, and also that $w \theta \in L$ if and only if $w \in L^{\prime}$.

ClaIM 3.1. The set $C=\{(\alpha, \alpha \theta): \alpha \in W\} \delta_{A^{\prime}}$ is regular in $A^{\prime}(2, \$)^{*}$.

We construct a non-deterministic finite automaton $M=\left(Q, A^{\prime}, \tau, s, F\right)$ accepting $C$, as follows:

$$
\begin{aligned}
Q= & \{s\} \cup\{(i, j): 1 \leq i, j \leq m\}, \quad F=\{(i, i): 1 \leq i \leq m\}, \\
\tau= & \left\{\left(s,\left(a_{p}, a_{p}\right), s\right): 1 \leq p \leq m\right\} \cup\left\{\left(s,\left(a_{p}, a_{q}\right),(p, q)\right): 1 \leq p, q \leq m\right\} \\
& \cup\left\{\left((p, q),\left(a_{i}^{\prime}, \$\right),(i, q)\right): 1 \leq i, p, q \leq m, p=i \eta\right\} .
\end{aligned}
$$

It is straightforward to prove that $M$ indeed accepts $C$. 
CLAIM 3.2. The set $D=\left\{(\alpha, \alpha \theta): \alpha \in L^{\prime}\right\} \delta_{A^{\prime}}$ is regular in $A^{\prime}(2, \$)^{*}$.

Note that

$$
D=\{(\alpha, \alpha \theta): \alpha \in W\} \delta_{A^{\prime}} \cap\left(\left(A^{\prime}\right)^{+} \times L\right) \delta_{A^{\prime}}
$$

The set $\{(\alpha, \alpha \theta): \alpha \in W\} \delta_{A^{\prime}}$ is regular by Claim 3.1 and the set $\left(\left(A^{\prime}\right)^{+} \times L\right) \delta_{A^{\prime}}$ is regular by [1, Proposition IIA 5.1]. It follows that $D$ is regular as the intersection of two regular sets.

\section{CLAIM 3.3. $L^{\prime}$ is regular.}

Let $\pi: A^{\prime}(2, \$)^{*} \rightarrow\left(A^{\prime}\right)^{*}$ be the homomorphism extending the map $(x, y) \mapsto x$, $(x, \$) \mapsto x,(\$, y) \mapsto \epsilon\left(x, y \in A^{\prime}\right)$. Then $L^{\prime}=D \pi$ is regular by Claim 3.2 since regular sets are closed under homomorphism.

CLAIM 3.4. $L_{x}^{\prime}$ is regular for every $x \in A^{\prime} \cup\{=\}$.

Since

$$
\left.L_{x}^{\prime}=\left\{(\alpha, \beta) \delta_{A^{\prime}}:\left(\exists \gamma, \gamma^{\prime}\right)\left((\alpha, \gamma) \delta_{A^{\prime}} \in D \& \ddot{(\gamma}, \gamma^{\prime}\right) \delta_{A^{\prime}} \in L_{x} \&\left(\gamma^{\prime}, \beta\right) \in D\right)\right\},
$$

it is regular by [3, Theorem 1.4.6], and the proof of the claim is complete.

We now proceed to complete the proof of part (i) of Theorem 1.1. By Claim 3.3 and Claim 3.4 we have that $\left(A^{\prime}, L^{\prime}\right)$ is an automatic structure for $S$. In the same way we may extend an automatic structure $(B, K)$ for $T$ to $\left(B^{\prime}, K^{\prime}\right)$. Without loss of generality we may assume that $A^{\prime} \cap B^{\prime}=\emptyset$; let $Y=A^{\prime} \cup B^{\prime}$.

Consider a new alphabet $X=A^{\prime} \times B^{\prime}$, and a new language

$$
J=\left\{\left(u_{1}, v_{1}\right) \cdots\left(u_{p}, v_{p}\right):\left(u_{i}, v_{i}\right) \in X, u_{1} \cdots u_{p} \in L^{\prime}, v_{1} \cdots v_{p} \in K^{\prime}\right\} .
$$

By [6, Theorem 2.1] it follows that $X$ is a generating set for $S \times T$ and that $J$ is mapped onto $S \times T$.

We now show that $(X, J)$ is an automatic structure for $S \times T$. First we note that the set $\left(L^{\prime} \times K^{\prime}\right) \delta_{Y}$ is regular in $Y(2, \$)^{*}$ by [1, Proposition IIA 5.1] and so $J=\left(L^{\prime} \times K^{\prime}\right) \delta_{Y} \cap X^{*}$ is regular.

Define two homomorphisms $\pi_{1}: X(2, \$)^{*} \rightarrow A^{\prime}(2, \$)^{*}$ and $\pi_{2}: X(2, \$)^{*} \rightarrow$ $B^{\prime}(2, \$)^{*}$ by

$$
\begin{aligned}
& \pi_{1}:\left(\left(u_{1}, v_{1}\right),\left(u_{2}, v_{2}\right)\right) \mapsto\left(u_{1}, u_{2}\right),\left(\left(u_{1}, v_{1}\right), \$\right) \mapsto\left(u_{1}, \$\right),\left(\$,\left(u_{2}, v_{2}\right)\right) \mapsto\left(\$, u_{2}\right), \\
& \pi_{2}:\left(\left(u_{1}, v_{1}\right),\left(u_{2}, v_{2}\right)\right) \mapsto\left(v_{1}, v_{2}\right),\left(\left(u_{1}, v_{1}\right), \$\right) \mapsto\left(v_{1}, \$\right),\left(\$,\left(u_{2}, v_{2}\right)\right) \mapsto\left(\$, v_{2}\right) .
\end{aligned}
$$


Note that

$$
\begin{aligned}
J_{=}= & \left\{\left((\alpha, \beta) \delta_{Y},(\gamma, \sigma) \delta_{Y}\right) \delta_{X}:(\alpha, \beta) \delta_{Y} \in J,(\gamma, \sigma) \delta_{Y} \in J,\right. \\
& \left.(\alpha, \gamma) \delta_{A^{\prime}} \in L_{=}^{\prime},(\beta, \sigma) \delta_{B^{\prime}} \in K_{=}^{\prime}\right\} \\
= & (J \times J) \delta_{X} \cap L_{=}^{\prime} \pi_{1}^{-1} \cap K_{=}^{\prime} \pi_{2}^{-1} .
\end{aligned}
$$

Since regular languages are closed under inverse homomorphism, $L_{==}^{\prime} \pi_{1}^{-1}$ and $K_{=}^{\prime} \pi_{2}^{-1}$ are regular, and so $J_{=}$is regular.

If $x=(u, v)$ with $u \in A^{\prime}$ and $v \in B^{\prime}$, then $L_{u}^{\prime}$ and $K_{v}^{\prime}$ are regular by Claim 3.4, and so

$$
J_{x}=(J \times J) \delta_{X} \cap L_{u}^{\prime} \pi_{1}^{-1} \cap K_{v}^{\prime} \pi_{2}^{-1}
$$

is regular. This completes the proof of part (i) of Theorem 1.1.

\section{One finite factor}

We now consider the direct product $S \times T$, where $S$ is finite with $S^{2}=S$, and $T$ is an arbitrary infinite automatic semigroup (not necessarily satisfying $T^{2}=T$ ). Let $S=\left\{s_{1}, \ldots, s_{m}\right\}$, and introduce a new alphabet $A=\left\{a_{1}, \ldots, a_{m}\right\}$, where $a_{i}$ represents $s_{i}$ for each $i$.

ClaIM 4.1. The set $C=\left\{\left(a_{i}, w\right): w \in A^{+}, w=a_{i}\right.$ in $\left.S\right\} \delta_{A}$ is regular in $A(2, \$)^{*}$.

Let $\pi: A^{+} \rightarrow S$ be the homomorphism extending the map $a_{i} \mapsto s_{i}$. Since $S$ is finite, $s_{i} \pi^{-1}$ is a regular set in $A^{+}$and so $\left(\left\{a_{i}\right\} \times s_{i} \pi^{-1}\right) \delta_{A}$ is regular in $A(2, \$)^{*}$. Now

$$
C=\bigcup_{1 \leq i \leq m}\left(\left\{a_{i}\right\} \times s_{i} \pi^{-1}\right) \delta_{A}
$$

is a finite union of regular languages, and hence is regular.

ClaIM 4.2. For any $x \in\left\{a_{1}, \ldots, a_{m}\right\} \cup\{\epsilon\}$ the set $D_{x}=\left\{(\alpha, \beta) \in A^{+} \times A^{+}\right.$: $\alpha x=\beta$ in $S\}$ is regular.

This follows immediately from the fact that

$$
D_{x}=\bigcup_{\substack{1 \leq i, j \leq m \\ s_{i} x=s_{j}}}\left(s_{i} \pi^{-1} \times s_{j} \pi^{-1}\right) \delta_{A} .
$$

We now let $T$ have an automatic structure $(B, K)$ and set $X=A \times B$. Let

$$
J=\left\{\left(u_{1}, v_{1}\right) \cdots\left(u_{p}, v_{p}\right):\left(u_{i}, v_{i}\right) \in X, v_{1} \cdots v_{p} \in K\right\}
$$


We show that $(X, J)$ is an automatic structure for $S \times T$.

If $(s, t) \in S \times T$, then $t=v_{1} \cdots v_{p} \in K$. Since $S^{2}=S$, there exist $u_{1}, \ldots, u_{p} \in A$ with $s=u_{1} \cdots u_{p}$, and so $(s, t)=\left(u_{1}, v_{1}\right) \cdots\left(u_{p}, v_{p}\right) \in J$. Therefore, $J$ maps onto $S \times T$. Let $Y=A \cup B$. Since

$$
J=\left(A^{+} \times K\right) \delta_{Y} \cap X^{*},
$$

$J$ is regular. We also have that

$$
J_{=}=(J \times J) \delta_{X} \cap D_{\epsilon} \pi_{1}^{-1} \cap K_{=} \pi_{2}^{-1}
$$

is regular, and that, for $x=(u, v) \in X$,

$$
J_{x}=(J \times J) \delta_{X} \cap D_{u} \pi_{1}^{-1} \cap K_{v} \pi_{2}^{-1}
$$

is regular, as required.

\section{Acknowledgement}

The fourth author would like to thank Hilary Craig for all her help and encouragement.

\section{References}

[1] G. Baumslag, S. M. Gersten, M. Shapiro and H. Short, 'Automatic groups and amalgams', J. Pure Appl. Algebra 76 (1991), 229-316.

[2] C. M. Campbell, E. F. Robertson, N. Ruškuc and R. M. Thomas, 'Automatic semigroups', Theoret. Comput. Sci., to appear.

[3] J. W. Cannon, D. B. A. Epstein, D. F. Holt, S. V. F. Levy, M. S. Paterson and W. P. Thurston, Word processing in groups (Jones and Bartlett, Boston, 1992).

[4] A. J. Duncan, E. F. Robertson and N. Ruškuc, 'Automatic monoids and change of generators', Math. Proc. Cambridge Philos. Soc. 127 (1999), 403-409.

[5] J. E. Hopcroft and J. D. Ullman, Introduction to automata theory, languages, and computation (Addison-Wesley, Reading, MA, 1979).

[6] E. F. Robertson, N. Ruškuc and J. Wiegold, 'Generators and relations of direct products of semigroups', Trans. Amer. Math. Soc. 350 (1998), 2665-2685.

Mathematical Institute

University of St Andrews

St Andrews KY16 9SS

Scotland

e-mail: cmc@st-andrews.ac.uk

edmund@dcs.st-andrews.ac.uk

nik@dcs.st-andrews.ac.uk
Department of Mathematics and Computer Science University of Leicester Leicester LE1 7RH England e-mail: rmt@mcs.le.ac.uk 